Recherches en didactique des langues et des cultures

Les cahiers de l'Acedle

$15-3 \mid 2018$

Quelles médiations en didactique des langues et des cultures?

\title{
Les manuels de FLE comme médiation en contexte chinois
}

Une étude comparative dans une perspective interculturelle

\section{Shuming Qiu}

\section{(2) OpenEdition}

\section{Journals}

Édition électronique

URL : http://journals.openedition.org/rdlc/3619

DOI : $10.4000 /$ rdlc.3619

ISSN : $1958-5772$

Éditeur

ACEDLE

\section{Référence électronique}

Shuming Qiu, «Les manuels de FLE comme médiation en contexte chinois », Recherches en didactique des langues et des cultures [En ligne], 15-3 | 2018, mis en ligne le 01 septembre 2018, consulté le 20 avril 2019. URL : http://journals.openedition.org/rdlc/3619 ; DOI : 10.4000/rdlc.3619

\section{Ce document a été généré automatiquement le 20 avril 2019.}

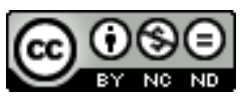

Recherches en didactique des langues et des cultures is licensed under a Creative Commons AttributionNonCommercial-NoDerivatives 4.0 International License 


\section{Les manuels de FLE comme médiation en contexte chinois}

Une étude comparative dans une perspective interculturelle

\section{Shuming Qiu}

\section{Introduction}

1 D'après Dervin \& Fracchiolla (2012: 10), le « renouvellement de la didactique de l'interculturel " porte sur la prise en conscience du "divers", du «complexe» ainsi que de " l'hétérogénéité du monde ». En ce sens, les manuels scolaires de langues étrangères, lieux de rencontre de diverses cultures - au moins la culture cible (en partie explicitée) et la culture des apprenants (explicite ou implicite) -, constituent une médiation interculturelle importante en didactique des langues.

Dans la situation chinoise, les manuels scolaires endossent une fonction considérable dans l'enseignement des langues étrangères au sein des établissements supérieurs. Étant donné que les manuels choisis par les universités doivent s'adapter aux objectifs du programme officiel élaboré par l'État, et que les examens nationaux, tels que le TFS $4^{1}$ et le TFS $8^{2}$ dans le cadre du FLE en Chine, sont " généralement basés sur les contenus enseignés d'après les manuels» (Huang, $2015: 14$ ), l'institution universitaire, les enseignants ainsi que les étudiants doivent s'appuyer sur les manuels scolaires. Une valeur d'«autorité » et de "sécurisation» est ainsi accordée aux manuels dans le cadre de l'enseignement des langues étrangères en contexte chinois (Ibid.). Plus généralement, les représentations proposées dans les manuels de langue étrangère (LE) sont «le plus souvent interprétées » par les apprenants, " comme spécifiques de cette culture et se transforment alors en exemples représentatifs, voire en modèles prototypiques" (Brancourt, 2009: 182). En raison de l'importance que l'on attache aux manuels de LE en Chine, les représentations de la culture cible construites dans ces manuels peuvent exercer des influences assez grandes sur les apprenants. Lorsque ces influences et la culture des étudiants se rencontrent lors de l'apprentissage à travers les manuels, la fonction de médiation des manuels pourra ainsi être mise en évidence. C'est en ce sens que nous avons constaté que le rôle de 
médiateur que joue un manuel de LE paraît particulièrement important dans le contexte de l'enseignement des langues étrangères en Chine.

Dans cette contribution, nous effectuerons une étude comparative des images de la France, mais aussi des représentations de l'identité et de l'altéritée ${ }^{3}$ dans des manuels de français importés de France et des manuels locaux en Chine, en prenant en compte leur complexité, leur hétérogénéité et leur diversité. À travers la comparaison, le questionnement principal de la recherche est : dans ces manuels, comment la France estelle représentée ? À travers quels supports ou références et comment ces représentations sont-elles interprétées, rapprochées et différenciées dans notre corpus étudié?

Le corpus se compose de 4 manuels parmi les plus répandus en Chine. D'un côté, les manuels importés Reflets (I, II) et Alter ego (I, II, III, IV). De l'autre, les manuels locaux Le français (I, II, III, IV) et Français (I, II, III, IV) ${ }^{4}$. Nous avons choisi les unités consacrées à la thématique de la situation des femmes dans ces manuels pour illustrer quelques-uns de nos questionnements. En premier lieu, parce que, d'après nos perceptions, la construction des représentations des femmes dans la société française et la société chinoise se distinguent l'une de l'autre. En deuxième lieu, la situation des femmes constitue de nos jours une thématique incontournable, de sorte qu'aucune société ne peut l'éluder. C'est pourquoi, d'après nous, il est intéressant de travailler sur cette thématique.

Dans le présent article, nous parlerons surtout d'un type d'activité que nous avons caractérisée comme "lieux de rencontre" pour voir comment la médiation est construite. Il s'agit des activités qui sont souvent mises en place à propos d'un document pédagogique présenté dans un manuel (par exemple, à propos d'un texte, d'une image, etc.), et qui visent à évoquer les réflexions personnelles des apprenants ou à rappeler des images sur le même sujet dans leur propre culture. Dans ces activités, les auteurs des manuels donnent la parole aux apprenants en leur demandant d'exprimer leurs propres points de vue ou de réfléchir sur leur propre culture en s'appuyant sur les représentations de la culture cible construites dans les manuels. Ces exercices constituent l'activité principale qui propose de thématiser explicitement ce qui relèverait d'une forme de rencontre entre la culture cible et la culture des apprenants. C'est la raison pour laquelle nous les avons définis comme « lieux de rencontre » dans notre travail ${ }^{5}$.

Citons ci-dessous des exercices des activités de «rencontre» dans Français comme exemple :

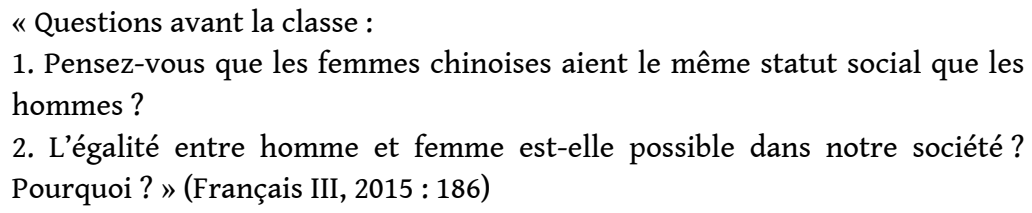

En réfléchissant sur ces questions, les étudiants sont sollicités à prendre conscience de leur identité culturelle lors de l'apprentissage de la culture cible, et ils sont conduits à réfléchir sur leur propre situation en Chine.

Ces activités paraissent assez fréquentes dans les manuels scolaires de LE, tandis que nous n'avons pas constaté de terme pédagogique spécifique pour les désigner. De ce fait, dans ce présent article, nous essayons de repérer ce type d'activités dans notre corpus en prenant en compte simultanément les deux critères suivants : premièrement, ces activités doivent être mises en avant à propos d'un document pédagogique présenté dans le manuel (souvent à propos d'un texte, d'une image, etc.); deuxièmement, ces activités 
doivent consister à évoquer les réflexions personnelles des apprenants ou à rappeler des images sur le même sujet dans la propre culture des apprenants. À partir de ces deux critères, nous pouvons faire un repérage de ces activités dans les unités consacrées à la situation des femmes dans les quatre manuels.

Dans cet article, nous explorerons dans la première partie la structure interne de l'activité « lieux de rencontre ». Dans la deuxième partie, nous comparerons précisément les manières de mettre en œuvre les activités de « rencontre » dans les unités consacrées à la thématique des femmes dans les quatre manuels. Des réflexions seront ensuite menées, dans la troisième partie, sur la construction des notions d'identité et d'altérité dans les histoires philosophiques française et chinoise pour enfin, dans la quatrième partie, tenter de découvrir les influences des contrastes philosophiques qui peuvent s'exercer, au moins partiellement, sur l'organisation des activités de « rencontre ».

\section{Structure interne de l'activité « lieux de rencontre »}

9 Cette activité comporte, selon les manuels, trois sortes d'exercices: «inciter à s'exprimer » « inciter à réfléchir sur sa propre culture » et « inciter à comparer » ${ }^{6}$.

\section{Premier type : « inciter à s'exprimer »}

10 L'«inciter à s'exprimer » consiste à poser une question qui conduit les étudiants à exprimer leurs propres points de vue à propos des représentations construites dans un manuel. Voici des exemples dans Le français, proposés après les textes qui parlent de l'inégalité entre les sexes :

«3.1) Que pensez-vous de l'émancipation des femmes?

8) Vous êtes pour ou contre l'idée de laisser les femmes sortir de la maison et trouver un emploi dans la société ? » (Le Français III, 2002 : 370)

Pour répondre à ces questions, les apprenants sont incités à énoncer leurs propres perceptions sur la question de « l'émancipation des femmes » et à prendre position sur la nécessité de chercher un emploi pour une femme. Voyons un autre exemple dans Alter ego , à propos des documents qui traitent la parité entre les sexes :

« 5. Formez des groupes dans la classe. Chaque groupe écrit un court article dans un journal, le 8 mars, pour faire part de sa position concernant la parité hommes/femmes. Dans votre texte, vous exprimez vos opinions et sentiments concernant la situation actuelle et à venir. » (Alter ego II, 2006 :

117)

Ce type d'exercice paraît ne pas être lié directement à la culture cible ni à la culture des étudiants : il ne leur est en effet explicitement demandé de réfléchir ni sur la culture cible ni sur la question dans le cadre de leur culture. Mais, en réalité, les opinions que proposent les étudiants peuvent impliquer des influences exercées par les représentations de la culture cible construites dans les documents pédagogiques présentés dans les manuels et aussi des points de vue (ou des imaginaires) que les étudiants se forment dans leur propre culture. C'est en ce sens que les représentations construites dans l'«inciter à s'exprimer» par les étudiants peuvent refléter leurs perceptions de la culture cible et/ou celles de leur propre culture. En effet, c'est d'abord une rencontre de la culture cible et de la culture des étudiants, puis une médiation entre 
ces deux parties qui ont contribué à construire les points de vue proposés par les étudiants dans ces exercices.

\section{Deuxième type : « inciter à réfléchir sur sa propre culture »}

11 Par rapport à l'«inciter à s'exprimer", ce deuxième type d'exercices évoque explicitement des termes qui visent la culture des étudiants. Dans les manuels importés qui sont plutôt des manuels de FLE généralistes et qui ne s'adressent pas notamment à un contexte culturel donné, on emploie des termes tels que "dans votre pays ", "dans votre culture », etc., comme l'indique un exercice écrit proposé dans Reflets :

«5. Texte libre.

Écrivez à un(e) ami(e) francophone pour donner votre point de vue sur la condition des femmes dans votre pays et pour lui expliquer comment sont réglés les rapports entre les sexes et pour quelles raisons. » (Reflets II, 2000 : 159)

12 En parallèle, les manuels locaux précisent plutôt le contexte des questions en employant des formulations telles que "dans notre pays », "dans notre société», "en Chine», etc. Citons un exemple dans Le Français :

«2. Comment est la situation actuelle des femmes en Chine? Pensez-vous que les femmes chinoises sont vraiment égales aux hommes?» (Le Français III, $2002: 370$ )

13 Par rapport à l'« inciter à s'exprimer » qui fait se rencontrer la culture cible et la culture des étudiants d'une manière plutôt implicite, l'« inciter à réfléchir sur sa propre culture » explicite la part de la culture des étudiants. Pourtant, il faut remarquer que l'« inciter à réfléchir sur sa propre culture » se limite plutôt à faire rappeler des images de la culture des apprenants, tandis que le troisième type d'exercices peut constituer un espace de rencontre de la culture cible et la culture des étudiants d'une manière plus explicite.

\section{Troisième type : « inciter à comparer »}

14 L'«inciter à comparer " demande non seulement aux apprenants de rappeler des représentations liées à leur propre pays, mais les invite aussi à comparer leur propre culture avec la culture cible : découvrir des différences, et même justifier quelle culture est préférable. En voici deux exemples proposés dans Alter ego :

«7. Comparez avec la répartition du travail chez vous : qui fait quoi à la maison? Combien d'heures par semaine ?» (Alter ego I, $2006: 79$ )

«9. Vous êtes une femme? Vous êtes un homme? Pour les tâches ménagères, vous préférez vivre en France ou dans votre pays? Justifiez. » (Alter ego I, $2006: 79$ )

Les étudiants sont ainsi sollicités à comparer la culture française et leur propre culture. C'est en ce sens que ce troisième type d'exercices peut inciter à une rencontre directe de ce qui relèverait de la culture cible et de la culture source des étudiants, et peut ainsi constituer un espace de médiation de la part d'identité et la part d'altérité avec une valeur de « concurrence » entre ces deux parts. 
Pour rendre plus précise la structure interne des activités de « rencontre ", nous avons aussi relevé le nombre de ces activités, en prenant compte le nombre de ces trois types d'exercices ainsi que le nombre total d'activités de "rencontre » dans les unités consacrées à la thématique des femmes dans les quatre manuels (tableau 1).

Tableau 1 - Nombre d'activités « lieux de rencontre » dans les unités consacrées à la thématique des femmes dans les quatre manuels

\begin{tabular}{|c|c|c|c|c|}
\hline & \multicolumn{2}{|l|}{ Manuels importés } & \multicolumn{2}{|c|}{ Manuels locaux } \\
\hline & Alter ego & Reflets & Le français & Français \\
\hline $\begin{array}{l}\text { nombre total } \\
\text { d'activités «lieux de } \\
\text { rencontre» }\end{array}$ & 15 & 2 & 10 & 5 \\
\hline $\begin{array}{l}\text { nombre total } \\
\text { d'activités du } \mathbf{1}^{\mathrm{er}} \\
\text { type : «inciter à } \\
\text { s'exprimer " }\end{array}$ & 7 & 1 & 8 & 3 \\
\hline $\begin{array}{lr}\text { nombre } & \text { total } \\
\text { d'activités du } \mathbf{2}^{\text {e }} \text { type } \\
: \text { «inciter à réfléchir } \\
\text { sur sa } \quad \text { propre } \\
\text { culture » }\end{array}$ & 4 & 1 & 2 & 2 \\
\hline $\begin{array}{lr}\text { nombre } & \text { total } \\
\text { d'activités du } \mathbf{3}^{\text {e }} & \text { type } \\
: & \text { "inciter } \\
\text { comparer }\end{array}$ & 4 & 0 & 0 & 0 \\
\hline $\begin{array}{l}\text { répartition des } \\
\text { activités dans une } \\
\text { (des) leçon(s) }\end{array}$ & $\begin{array}{l}\text { dispersées dans } \\
\text { une leçon d'une } \\
\text { manière assez } \\
\text { équilibrée }\end{array}$ & $\begin{array}{l}\text { dispersées dans } \\
\text { une leçon d'une } \\
\text { manière assez } \\
\text { équilibrée }\end{array}$ & $\begin{array}{ll}\text { regroupées } \\
\text { dans } & \text { la } \\
\text { rubrique de } \\
\text { textes }\end{array}$ & $\begin{array}{l}\text { regroupées } \\
\text { principalement } \\
\text { dans la rubrique de } \\
\text { textes }\end{array}$ \\
\hline $\begin{array}{l}\text { documents en marge } \\
\text { desquels } \\
\text { apparaissent ces } \\
\text { activités }\end{array}$ & $\begin{array}{l}\text { texte, } \\
\text { illustration, } \\
\text { tableau, test, } \\
\text { caricature }\end{array}$ & texte, tableau & texte & $\begin{array}{l}\text { texte, exercice de } \\
\text { compréhension } \\
\text { orale }\end{array}$ \\
\hline
\end{tabular}

À partir de ce tableau, nous explorerons dans la partie suivante les manières d'organiser ces activités dans notre corpus.

\section{Analyses du corpus}

Dans cette partie, nous avons choisi de regarder plus en détail comment ces activités sont organisées dans les unités consacrées à la situation des femmes dans les quatre manuels en suivant les quatre axes d'analyse : est-ce que l'accent est mis sur ces activités « lieux de rencontre »? Est-ce que les activités « lieux de rencontre » demandent aux étudiants de comparer explicitement la culture cible et leur propre culture? Les activités « lieux de rencontre » sont-elles proposées avant ou après un document pédagogique qui construit 
des représentations de la culture cible ? Est-ce que la manière de poser une question dans les activités « lieux de rencontre » traduit un présupposé explicite?

\section{Premier axe d'analyse : est-ce que l'accent est mis sur les activités « lieux de rencontre »?}

En premier lieu, la proportion de ces activités est assez élevée dans les manuels importés. Pour rendre cette question plus claire, nous examinerons le nombre de pages de la (des) leçon(s) qui aborde(nt) particulièrement la situation des femmes dans les quatre manuels et le nombre d'activités «lieux de rencontre» dans ces leçons pour comparer la proportion de ces activités dans les leçons consacrées à la problématique des femmes dans ces manuels (tableau 2).

Tableau 2 - Proportion des activités « lieux de rencontre » dans les unités consacrées à la situation des femmes dans les quatre manuels

\begin{tabular}{|l|l|l|l|l|}
\hline \multicolumn{2}{|l|}{$\begin{array}{l}\text { Manuels } \\
\text { importés }\end{array}$} & \multicolumn{2}{l|}{ Manuels locaux } \\
\cline { 2 - 6 } & Alter ego & Reflets & $\begin{array}{l}\text { Le } \\
\text { français }\end{array}$ & Français \\
\hline $\begin{array}{l}\text { nombre de pages de la (des) leçon(s) consacrée(s) à } \\
\text { la situation des femmes }\end{array}$ & 26 & 2 & 33 & 27 \\
\hline $\begin{array}{l}\text { nombre d'activités « lieux de rencontre » dans ces } \\
\text { leçons }\end{array}$ & 15 & 2 & 10 & 5 \\
\hline $\begin{array}{l}\text { proportion de ces activités dans ces leçons } \\
\text { (activité par page) }\end{array}$ & $\begin{array}{l}15 / 26 \\
(0,58 / \\
\text { page })\end{array}$ & $\begin{array}{l}2 / 2 \\
(1 / \\
\text { page })\end{array}$ & $\begin{array}{l}0,30 / \\
\text { page })\end{array}$ & $\begin{array}{l}(0,19 / \\
\text { page })\end{array}$ \\
\hline
\end{tabular}

Nous pouvons constater à partir de ce tableau que la proportion dans les deux manuels importés dépasse 0,50/page, et que celle dans Reflets atteint même une activité par page, bien que le corpus dans Reflets soit limité. En parallèle, la proportion dans les deux manuels locaux est beaucoup moindre.

De plus, ces activités sont assez largement répandues dans les manuels importés. Elles sont proposées d'une manière assez équilibrée dans plusieurs rubriques pédagogiques dans une leçon, telles que la rubrique de textes, la rubrique d'exercices, la rubrique d'illustration, etc., tandis que dans les manuels locaux, elles sont principalement présentées dans la rubrique de textes (cf. tableau 1).

Enfin, dans les manuels importés, ces activités sont mises en lien avec diverses formes de documents, par exemple texte, illustration, tableau, test, caricature dans Alter ego ainsi que texte et tableau dans Reflets; alors que dans les manuels locaux, elles sont plutôt associées aux textes ( $c f$. tableau 1$)$.

21 Pour conclure, nous pouvons en déduire que les manuels importés ont relativement plus mis l'accent sur les activités « lieux de rencontre » par rapport aux manuels locaux. 


\section{Deuxième axe d'analyse : est-ce que les activités « lieux de rencontre » demandent aux étudiants de comparer explicitement la culture cible et leur propre culture?}

À partir du tableau 1, nous pouvons constater que Reflets, Le français et Français ne proposent pas le troisième type d'exercices «inciter à comparer » dans les unités consacrées à la situation des femmes. Ces trois manuels se contentent de faire réfléchir les étudiants, potentiellement, sur leur situation, comme l'indique l'exemple suivant dans Le Français :

«3. 2) Comment est la situation actuelle des femmes en Chine? Pensez-vous que les femmes chinoises sont vraiment égales aux hommes?» (Le Français II, $2002: 370$ )

Seul Alter ego aborde le troisième type d'exercices et incite les apprenants à comparer d'une manière explicite la culture cible et leur propre culture. En voici quelques exemples :

«7. Comparez avec la répartition du travail chez vous : qui fait quoi à la maison ? Combien d'heures par semaine ?» (Alter ego I, $2006: 79$ )

«10. Prenez connaissance des chiffres (montrés dans les tableaux "les dix métiers les plus féminisés" et "les dix métiers les moins féminisés") et comparez avec la situation dans votre pays. » (Alter ego II, 2006 : 99)

La consigne "comparer» dans ces questions peut conduire les étudiants à mettre en relation directement leur propre situation et la situation en France, puis à découvrir des ressemblances et des différences entre les deux cultures. La part d'identité et celle d'altérité sont ainsi mises en rencontre explicitement, voire opposées.

En outre, nous avons également remarqué que quelques exercices vont encore plus loin. Ces exercices visent à inciter les apprenants à prendre une position entre la culture cible et leur culture, en indiquant une préférence :

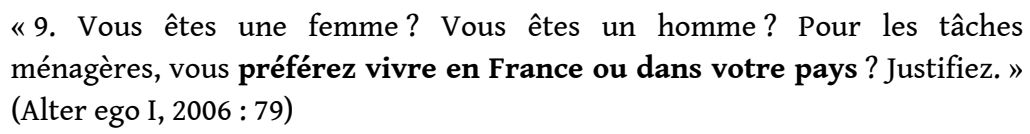

Pour répondre à cette question, il ne suffit pas de trouver des points communs et des différences. Les apprenants sont invités à faire un choix entre la culture cible et leur propre culture, soit l'une soit l'autre. Cela part du présupposé que la situation en France et celle dans la culture des étudiants sont nécessairement différentes et on les enjoint à prendre parti pour ou contre l'une ou l'autre, ce qui semble opposé à une perspective de médiation interculturelle.

\section{Troisième axe d'analyse : les activités « lieux de rencontre » sont- elles proposées avant ou après un document pédagogique qui construit des représentations de la culture cible?}

Dans les manuels importés et Le français, les activités de "rencontre " sont souvent proposées après un document pédagogique (un texte, par exemple) qui met en avant des représentations de la France, tandis que Français préfère inciter d'abord les apprenants à 
réfléchir sur la situation en Chine, puis aborder la situation française dans les textes, et enfin inviter les étudiants à proposer leurs points de vue sur le phénomène abordé dans les textes. Dans Français, nous pouvons remarquer dans la plupart des leçons une rubrique "questions avant la classe", abordée avant la lecture des textes, comme sensibilisation, pour poser des questions du type « inciter à réfléchir sur sa propre culture » :

"Questions avant la classe :

1. Pensez-vous que les femmes chinoises aient le même statut social que les hommes?

2. L'égalité entre homme et femme est-elle possible dans notre société ? Pourquoi ? (Français III, $2015: 186$ )

Ainsi, avant d'aborder la culture cible, les apprenants peuvent-ils construire quelques représentations de leur propre culture.

Ensuite, un texte évoque une femme responsable de la CFDT et les difficultés qu'elle a rencontrées, d'une part dans sa vie professionnelle en tant que femme, et, de l'autre dans sa recherche d'équilibre entre travail et vie familiale. À la suite du texte, quelques exercices du type « inciter à s'exprimer » sont mis en avant :

\section{«II. Activités :}

1. Pensez-vous qu'une femme puisse trouver un parfait équilibre entre sa carrière et sa vie familiale? Si oui, citez l'exemple d'un cas réussi ; si non, quels sont les handicaps d'après vous?

2. Parmi les femmes suivantes, laquelle serait votre idole? Justifiez votre affirmation. Angela Merkel - Wu Yi - Christine Lagarde - Laura Bush - Gloria Arroyo - Céline Dion - Yang Lan - Zhang Manyu - Li Qingzhao - Simone de Beauvoir. » (Français III, 2012 : 192)

Le premier exercice, qui invite les étudiants à construire leurs propres opinions à propos des représentations construites dans le texte, aborde des difficultés qu'une femme engagée dans la vie syndicale française a rencontrées pour équilibrer sa carrière et sa vie familiale.

Le second exercice incite les apprenants à choisir un modèle féminin idéal («idole »). Cet exercice peut conduire à réfléchir à quel type de femme les étudiantes veulent ressembler et, pour des étudiants, à réfléchir au type de femme qu'ils apprécient davantage. Cela peut effectivement contribuer à construire leur propre identité. Par ailleurs, les femmes montrées dans le deuxième exercice sont de grands personnages féminins qui ont tous réussi leur vie professionnelle. Elles sont de diverses professions (femme politique, intellectuelle et actrice), de diverses nationalités (française, chinoise, allemande, américaine, philippine et canadienne) et de diverses situations familiales (mariée, divorcée et célibataire). Il faut remarquer que la situation familiale d'une femme peut également impliquer dans une certaine mesure la question de l'équilibre entre carrière et vie familiale : le statut marital constitue un des critères importants pour prendre comme modèle une des personnalités proposées. Cela montre que le second exercice renvoie également dans une certaine mesure au texte.

En somme, à partir de ces analyses sur l'organisation des activités de "rencontre " proposées dans Français, nous pouvons remarquer que, à la différence des trois autres manuels, les activités de "rencontre" présentées dans Français sont organisées en suivant davantage les trois démarches suivantes. 

peut conduire les étudiants à formuler une réponse attendue par l'auteur du manuel. Par exemple, après les textes qui parlent de l'inégalité entre les sexes, nous avons trouvé cette question :

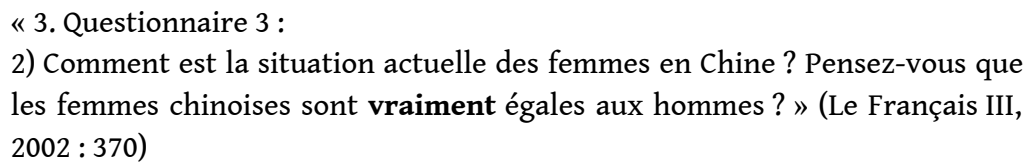

L'adverbe "vraiment " attribue une valeur de vérité absolue à cette question. Cela peut conduire les étudiants à évoquer un maximum de possibilités pour vérifier si une égalité absolue existe entre les sexes. Il faut admettre que, dans le monde actuel, il est difficile pour les femmes de bénéficier d'une position sociale tout à fait identique aux hommes. En ce sens, cette question risque probablement d'entraîner une réponse négative de la part des étudiants. Alors, après avoir critiqué l'inégalité entre les sexes en France dans les textes, le rédacteur peut ainsi conduire les étudiants à évoquer une situation semblable dans leur propre culture. Par contre, nous n'avons pas constaté d'exemples de présupposé explicite dans d'autres manuels.

Ce rapport à l'identité et à l'altérité qui transparaît de façon contrastée entre les différents manuels à travers la réalisation des activités « lieux de rencontre » incite à réfléchir à ce qui peut contribuer, pour les apprenants à construire des représentations de façon différente. 


\section{Réflexions sur des contrastes philosophiques}

de trouver quelques éléments de réponse, dans un premier temps, en explorant la construction des notions d'identité et d'altérité dans les histoires philosophiques française et chinoise. En effet, elles ne renvoient pas aux mêmes présupposés.

\section{Étapes « identité vs altérité » et « post identité vs altérité » en philosophie française}

Les recherches mobilisant les notions d'identité et d'altérité ont connu de nombreux développements pendant des siècles en France. Des philosophes chinois contemporains tels que Zhang (2002) et Lin (2010) ont essayé de les résumer très brièvement en deux étapes.

La première étape consiste à mettre en contradiction l'identité et l'altérité. D'un côté, des philosophes tels que Descartes (2013), Sartre (1976) ont insisté plutôt sur l'identité. Le cogito de Descartes marque la fondation du solipsisme moderne. Le cogito cartésien met en avant l'ego en tant que sujet qui pense et qui doute. Au sein même du doute, le sujet affirme sa valeur en négligeant celle de l'autre. L'altérité est ainsi déductible du cogito. Le cogito cartésien est souvent censé être trop réflexif et introspectif pour pouvoir s'ouvrir à l'autre et pour percevoir la valeur de celui-ci (cf. Ayissi, 2005 ; Courtine-Denamy, 2007). L'ontologie phénoménologique de Sartre n'échappe pas non plus au solipsisme. L'existence de l'identité pèse considérablement sur celle de l'autre. La relation entre l'identité et l'altérité est plutôt conflictuelle, et l'irruption de l'altérité dans l'identité prédispose à l'affrontement. Cette phobie de l'altérité sartrienne vise davantage à diaboliser des formes de l'autre (cf. Ayissi, 2005). D'un autre côté, Levinas (2014) a vivement mis en cause le cogito cartésien et l'ontologie phénoménologique sartrienne. Il insiste sur des formes d'altérité absolue, et il met en exergue une relation asymétrique entre l'identité et l'altérité : la première doit être responsable de la seconde, mais le contraire n'est pas nécessaire. Ce qu'il met en valeur, c'est plutôt un humanisme pour autrui.

41 Étant donné que les recherches portant sur les notions d'identité et d'altérité dans cette étape en philosophie française tendent davantage à opposer la notion d'identité à celle d'altérité, des philosophes chinois contemporains ont essayé de caractériser très brièvement cette étape comme étape «identité vs altérité " (Zhang, 2002: 3-14; Lin, $2010: 8-9$ ).

Dans une seconde étape, des philosophes tels que Ricœur (1990) et Todorov (1982) ont proposé un pouvoir équivalent et une relation réciproque entre l'identité et l'altérité. Ricœur critique la position de Levinas qui a trop insisté sur le primat de l'autre, alors que Ricœur emploie le soi qui se distingue de l'ego non réfléchi cartésien (je) pour désigner l'ipséité. Pour Ricœur, l'autre n'est pas seulement "la contrepartie du même ", mais " appartient à la constitution intime de son sens ", et donne ainsi sa force à l'expression : " soimême comme un autre» (Ricœur, 1990: 380, 378). À la différence de Levinas, Ricœur attribue un pouvoir équivalent au soi et à l'autre. Todorov propose également que l'altérité de l'autre doive être vue équivalente à l'identité du moi. Il critique le moi 
ignorant l'altérité d'autrui : même si le moi prétend aimer et prendre soin de ce dernier, son seul but ne se trouve que dans la volonté de transformer l'autre au nom du moi, donc de le soumettre. Il propose ainsi pour sa part «une nouvelle variante de l'amour porté à autrui », un amour "non plus assimilationniste mais distributif: chacun a ses propres valeurs » (Todorov, 1982 : 196). Il appelle cette nouvelle position le « perspectivisme» (Ibid., 195).

Des philosophes chinois contemporains ont qualifié cette seconde étape comme "post identité vs altérité » (Zhang; Lin, ibid.), parce que les concepts philosophiques dans cette étape proposant un pouvoir équivalent à l'identité et à l'altérité ont été réalisés à l'époque contemporaine après des critiques envers les points de vue de la première étape, qui mettaient en exergue une contradiction identité-altérité.

\section{Étape « anté identité vs altérité » en philosophie chinoise et le wo en taoïsme}

À la différence des études développées et diversifiées en philosophie française, la philosophie chinoise n'a presque jamais traité spécifiquement les notions d'identité et d'altérité. En effet, l'histoire philosophique chinoise évite de distinguer les deux. C'est pourquoi des philosophes chinois contemporains considèrent la philosophie chinoise comme "anté identité vs altérité » (Zhang; Lin, ibid.) : la tradition philosophique chinoise n'a jamais connu d'étape de "dualité » dite "identité vs altérité » et elle insiste plutôt sur l'idée que tous les éléments de l'univers sont liés étroitement les uns aux autres pour construire une harmonie en un tout.

Nous nous demandons donc vers quelle direction est destiné ou s'unifie ce "tout ». Le taoïsme l'a renommé yi (homogénéité) : "Le tiandi (le ciel et la terre) coexiste et s'entrecroise avec le wo (moi), et tous les éléments s'unifient en wo.» (Sun, $2007: 4$ ) En ce sens, le taoïsme axe plutôt le yi (homogénéité) sur le wo (moi). Et celui-ci constitue son point de départ et sa perspective pour connaître le monde. La tradition philosophique chinoise considère ainsi les points de vue du wo comme idées partagées qui peuvent naturellement s'appliquer au wanwu ${ }^{9}$ (y compris le bieren ${ }^{10}$ ).

Jullien a aussi constaté ce principe philosophique chinois, et il a essayé de l'expliquer à sa manière. D'après lui, étant donné que la culture chinoise croit "pouvoir s'étendre indéfiniment sur le monde habité... sans rencontrer la résistance d'une véritable altérité et du seul fait de sa puissance institutionnelle, morale et politique ", et qu'elle "ne connaît de Récit que celui de sa propre histoire ", pour elle, le monde doit ainsi obéir naturellement aux «règles constantes» que la culture chinoise possède « intrinsèquement » (Jullien, 2008 : 123-124). C'est pourquoi c'est plutôt un « non-soupçon d'une altérité (extériorité) possible » qui domine dans la philosophie chinoise et la culture chinoise « se perçoit une culture de la globalité qu'elle tient d'emblée pour une évidence acquise » (ibid. : 120-121). En ce sens, pour Jullien, l'universalité culturelle de la Chine « va de soi », et la Chine "n'a même pas à se poser la question de leur universalité possible » (ibid. : 120).

Ainsi, les recherches portant sur l'identité et l'altérité en philosophie chinoise n'ont-elles pas connu une "vraie révolution» jusqu'à présent. La plupart des recherches ne constituent que des explications ou des critiques sur d'anciens travaux. 


\section{Une perspective contrastive}

La relation entre le moi et l'autre en philosophie française et celle entre le wo et le wanwu en philosophie chinoise diffèrent alors l'une de l'autre. Une différence remarquable entre elles se trouve dans le fait que la philosophie française vise plutôt à séparer la part d'identité et celle d'altérité, alors qu'en philosophie chinoise le wanwu est inclus en wo, et rien n'est complètement séparé. Nous essayerons de préciser ces caractéristiques dans le tableau suivant (Figure 1).

Figure 1 - Caractéristiques des trois étapes en philosophie française et en philosophie chinoise

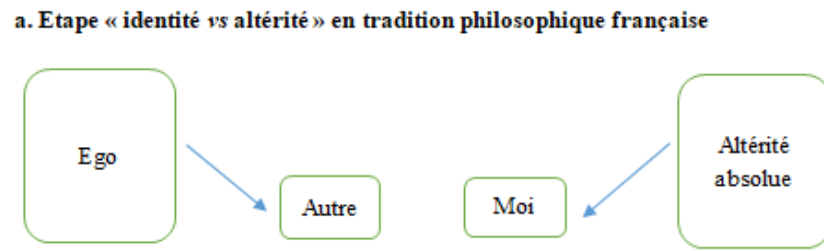

b. Etape «post identité vs altérité » en philosophie contemporaine française

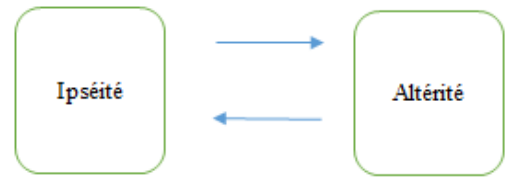

c. Etape «anté identité vs altérité » en tradition philosophique chinoise

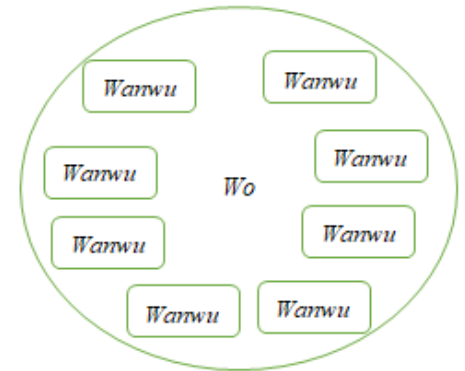

Ce tableau peut dans une certaine mesure témoigner des caractéristiques de la construction des notions d'identité et d'altérité en philosophie française et en philosophie chinoise. Dans la partie suivante, nous tenterons d'explorer l'influence de ces contrastes philosophiques qui peuvent s'exercer sur l'organisation des activités « lieux de rencontre » dans notre corpus.

4. L'organisation des activités « lieux de rencontre" et l'influence des contrastes philosophiques

Les activités didactiques « lieux de rencontre » présentées dans les manuels peuvent être interprétées, au moins partiellement, à la lumière de ces contrastes philosophiques.

Pour commencer, à partir des analyses sur notre corpus, nous remarquons que les manuels importés mettent davantage l'accent sur les activités de "rencontre». Cela traduit qu'ils ont non seulement «choisi » des représentations pour les montrer aux apprenants considérés comme autres, en mettant ceux-ci dans une position de «réception»; mais ils ont aussi choisi de faire prendre position à ces apprenants, en conduisant ceux-ci à réfléchir et à exprimer leurs propres opinions à propos des 
représentations construites dans les manuels. Ainsi, un pouvoir de construire des représentations, comme les rédacteurs peuvent le faire, est-il attribué aux étudiants. C'est en ce sens que les manuels importés ont accordé une forme de pouvoir équivalent aux apprenants. Cela pourrait, dans une certaine mesure, renvoyer aux concepts présentés en philosophie contemporaine française qui propose un pouvoir équivalent entre l'identité et l'altérité.

Ensuite, à partir des analyses sur notre corpus dans Alter ego, nous pouvons constater que des activités «lieux de rencontre » demandent aux étudiants de comparer la situation dans la culture cible et celle dans leur propre culture, et même de prendre une position entre ces deux situations dans les deux cultures, soit l'une soit l'autre. Cela part du postulat que la situation dans la culture cible et celle dans la culture des étudiants sont forcément séparées et se distinguent l'une de l'autre. Et cela pourrait correspondre à un principe en philosophie française qui vise à séparer ou à différencier la part de l'identité de celle de l'altérité.

Dans notre corpus dans Français, des activités de "rencontre » sont organisées devant les textes pour inciter d'abord les apprenants à parler de la situation en Chine; ensuite la situation en France est abordée dans les textes; et enfin d'autres activités de «rencontre " sont présentées pour demander aux étudiants de montrer leurs propres opinions sur la question traitée. Ainsi, pour les étudiants, la perspective de la situation chinoise (wo) constitue-t-elle un point de départ pour découvrir ensuite l'altérité de la situation française ; et une visée didactique consiste-t-elle enfin en construction de leur propre identité culturelle, aussi sous forme du wo. De ce fait, la part du wo joue toujours un rôle essentiel dans l'organisation des activités « lieux de rencontre » dans notre corpus dans Français. Cela pourrait, dans une certaine mesure, refléter les principes du wo en taoïsme.

55 Enfin, une présupposition est traduite par la manière d'organiser une question dans les activités «lieux de rencontre» dans notre corpus dans Le français. Comme nous avons parlé ci-dessus, ce présupposé peut conduire les étudiants à évoquer que le phénomène de l'inégalité entre les femmes et les hommes est une situation partagée en France et en Chine. Cela pourrait correspondre au principe du yi (homogénéité) selon lequel des valeurs et des phénomènes sont partagés dans le monde en tradition philosophique chinoise. En ce sens, ce principe du yi pourrait, dans une certaine mesure, exercer des influences sur la rédaction du manuel local chinois.

\section{Conclusion}

Ces éclairages philosophiques permettent de commencer à interpréter les analyses effectuées à partir des manuels. Mais il faudra aussi avoir recours à d'autres sources pour nourrir et approfondir ces interprétations, esquissées seulement à partir d'un corpus réduit. Nous examinerons notamment, dans des travaux ultérieurs, l'influence d'autres éléments, comme les idéologies dominantes dans les deux pays, ainsi que les caractéristiques des auteurs des manuels concernés (âge, sexe, histoire et expérience, positionnement, etc.).

Les manuels scolaires de FLE jouent un rôle de médiateur considérable dans l'enseignement du français au sein des établissements supérieurs en contexte chinois. Les activités « lieux de rencontre » identifiées dans les manuels incitent à faire se rencontrer, 
soit implicitement soit explicitement, des représentations de la culture cible et celles issues de la culture des étudiants. Ces activités peuvent ainsi mettre en relief la fonction de médiation: la part d'identité et celle d'altérité peuvent davantage intervenir et se coordonner. Ce qui peut enfin contribuer à la construction de la propre identité interculturelle des apprenants.

\section{BIBLIOGRAPHIE}

Ayissi, L. (2005). « Cogito et Altérité ». In Wanguè, T. B. (dir.). L'individuel et le collectif. Marne : Dianoïa.

Berthet, A., Hugot C., Kizirian, V., Sampsonis, B. \& Waendendries, M. (2006). Alter ego. Paris : Hachette.

Brancourt, V. (2009). « Comptes rendus de lecture : French Studies in Southern Africa : études françaises en Afrique australe ». Revue japonaise de didactique du français, vol. 4, $\mathrm{n}^{\circ}$ 2. pp. 182-184.

Capelle, G., Gidon, N., Qi, Y. Z. \& Zhou, Z. C. (2007). Reflets (version adaptée). Pékin : Maison d'édition de l'enseignement et des recherches des langues étrangères.

Cao, D. M. (2015). Français. Shanghai : Maison d'édition de l'éducation des langues étrangères de Shanghai.

Dervin, F. \& Fracchiolla, B. (dir.) (2012). Anthropologies, interculturalité et enseignement-apprentissage des langues. Berne : P. Lang.

Descartes, R. (1637 [2016]). Discours de la méthode. Paris : Flammarion.

Dong, Y. Y. (2012). « Analyse du TFS4 et sa place dans le curriculum chinois ». Synergies Chine, $\mathrm{n}$ 6. 6p. 81-95.

Jullien, F. (2008). De l'universel : De l'uniforme, du commun et du dialogue entre les cultures. Paris : Fayard.

Encyclopcedia Universalis (2007). Consulté en août 2017 : http://www.universalis.fr/encyclopedie/ alterite-philosophie.

Huang, L. (2015). Médiation culturelle et manuel de FLE : Altérité et identité dans Le français. Canton : Université Sun Yat-sen.

Levinas, E. (2014). Le temps et l'autre. Paris : PUF.

Lin, K. J. (2010). Homogénéité du Ciel et de l'humain vs Dualité de l'identité et l'altérité: Une perspective importante pour comparer la tradition philosophique chinoise et la tradition philosophique occidentale. Pékin : Maison d'édition des sciences sociales et des documents historiques. (林可济, 2010, “天 人合一”与“主客二分”--中西哲学比较的重要视角, 北京 : 社会文献科学出版社。).

Ma, X. H. (2002). Le français. Pékin : Maison d'édition de l'enseignement et des recherches des langues étrangères.

Ricœur, P. (1990). Soi-même comme un autre. Paris : Le Seuil.

Sartre, J.-P. (1976). L'Être et le néant. Paris : Gallimard. 
Sun, T. H. (2007). Tchouang Tseu. Pékin : Maison d'édition de la nation chinoise. (孙通海, 2007, 庄子, 北京 : 中华书局。).

Todorov, T. (1982). La conquête de l'Amérique : la question de l'autre. Paris : Seuil.

Zhang, S. Y. (2002). Introduction à la philosophie. Pékin : Maison d'édition de l'Université de Pékin. (张世英, 2002, 哲学导论, 北京: 北京大学出版社。).

\section{NOTES}

1. TFS4 : Test national de français comme spécialité niveau 4, destiné aux «étudiants spécialisés en études françaises à la fin de leur $2^{\mathrm{e}}$ année », est organisé chaque année en mai par "la Commission nationale d'examens des langues étrangères du ministère de l'Éducation et l'Association chinoise des professeurs de français ", " en vue d'évaluer le niveau de français des étudiants du cycle de base universitaire » (Dong, $2012: 81$ ).

2. TFS 8 : Test national de français comme spécialité niveau 8 , destiné aux étudiants spécialisés en études françaises dans leur $4^{\mathrm{e}}$ année, est organisé chaque année en mars également par la Commission nationale d'examens des langues étrangères du ministère de l'Éducation et l'Association chinoise des professeurs de français, en vue d'évaluer le niveau de français des étudiants qui ont appris le français comme spécialité depuis presque quatre ans.

3. Les représentations de l'identité portent ici notamment des images de la France construites dans les manuels importés de France, alors que pour les représentations de l'altérité, il s'agit ici des images de la France construites dans les manuels locaux en Chine.

4. Ces manuels sont destinés aux étudiants de français dans leurs deux premières années à l'université en Chine. Reflets I est de niveaux A1/A2, Reflets II est de niveaux B1/B2 ; Alter ego I est de niveau A1, Alter ego II est de niveau A2, Alter ego III est de niveau B1, Alter ego IV est de niveau B2 ; Le français I est plutôt de niveau A1, Le français II est plutôt de niveau A2, Le français III est plutôt de niveau B1, Le français IV est plutôt de niveau B2 ; Français I est plutôt de niveau A1, Français II est plutôt de niveau A2, Français III est plutôt de niveau B1, Français IV est plutôt de niveau B2. Pour les deux manuels locaux, Français et Le français, les rédacteurs n'ont jamais indiqué leur niveau du CECRL. Le classement a été réalisé ici par l'auteur de l'article en suivant des expériences d'enseignement.

5. Nous n'avons pas remarqué de terme spécifique pour désigner ce type d'activité dans les manuels, et « lieux de rencontre » n'est pas une vraie citation repérée de notre corpus. C'est une dénotation que nous avons proposée à ces activités.

6. « Inciter à s'exprimer » « inciter à réfléchir sur sa propre culture » et « inciter à comparer » ne sont pas non plus de vraies citations dans les manuels. Ce sont toujours des dénotations attribuées par l'auteur de l'article.

7. Le wo est le pinyin du moi, et il est employé ici pour se distinguer du moi en philosophie française.

8. Le tiandi (le ciel et la terre) représente le wanwu (tous les êtres et tous les objets dans le monde).

9. Le wanwu signifie tous les êtres et tous les objets dans le monde.

10. Le bieren est le pinyin chinois qui signifie autrui. 


\section{RÉSUMÉS}

Les manuels scolaires de langues étrangères sont un espace de rencontre de la culture cible et de la propre culture des apprenants. C'est en ce sens qu'ils constituent une médiation interculturelle essentielle en didactique des langues. Ce rôle de médiateur paraît surtout important en contexte chinois. C'est pourquoi nous mènerons un travail de recherche comparatif des représentations de la France et aussi de la Chine dans des manuels de français importés de France et des manuels locaux en Chine. Ce présent article portera en particulier sur un type d'activités que nous avons caractérisées comme «lieux de rencontre " pour analyser comment cette médiation est construite et pour débuter une interprétation de la part interculturelle de ces activités, en prenant en compte l'influence de certains contrastes philosophiques entre la France et la Chine.

外语教材能体现目标语文化与学生的母文化之交汇, 所以外语教材在外语教学中是一种重要 的跨文化中介。这种中介作用在中国的外语教学中扮演着尤为重要的角色。因此我们将对从 法国引进的法语教材与在中国本土编写的法语教材中有关法国与中国的表征作比较研究。本 文侧重讨论一种我们定义为 “lieux de rencontre” 的练习，用以探讨这种中介作用是如何体现 的, 并依托中法哲学观点的差异来分析这种练习。

\section{INDEX}

Mots-clés : médiation, étude comparative, étude interculturelle, manuels de FLE, activités «lieux de rencontre»

\section{AUTEUR}

\section{SHUMING QIU}

E.A. 4428 DYNADIV, Université de Tours

Shuming QIU est doctorante en didactique du FLE (département des sciences du langage) à l'Université de Tours, sous la direction de Véronique Castellotti. Elle est rattachée à l'équipe E.A. 4428 DYNADIV. Elle s'intéresse notamment aux recherches interculturelles sur la construction des représentations dans les manuels scolaires de FLE. Elle a également été enseignante de FLE à l'université en Chine.

shuming.qiu[at]etu.univ-tours.fr 